\title{
BMJ Open Cohort profile: HealthWise Wales. A research register and population health data platform with linkage to National Health Service data sets in Wales
}

\author{
Lisa Hurt (D , , Pauline Ashfield-Watt, ${ }^{1}$ Julia Townson, ${ }^{2}$ Luke Heslop, ${ }^{1}$ \\ Lauren Copeland, ${ }^{1}$ Mark D Atkinson, ${ }^{3}$ Jeffrey Horton, ${ }^{4}$ Shantini Paranjothy ${ }^{1}$
}

To cite: Hurt L, Ashfield-Watt P, Townson J, et al. Cohort profile: HealthWise Wales. A research register and population health data platform with linkage to National Health Service data sets in Wales. BMJ Open 2019;9:e031705. doi:10.1136/ bmjopen-2019-031705

- Prepublication history for this paper is available online. To view these files, please visit the journal online (http://dx.doi org/10.1136/bmjopen-2019031705).

Received 15 May 2019 Revised 01 August 2019 Accepted 17 October 2019

Check for updates

(c) Author(s) (or their employer(s)) 2019. Re-use permitted under CC BY-NC. No commercial re-use. See rights and permissions. Published by BMJ.

${ }^{1}$ Division of Population Medicine, Cardiff University School of

Medicine, Cardiff, UK

${ }^{2}$ Centre for Trials Research, Cardiff University, Cardiff, UK ${ }^{3}$ Medical School, Swansea University, Swansea, UK

${ }^{4}$ Patient and Public

Representative, Cardiff

University, Cardiff, UK

Correspondence to

Professor Shantini Paranjothy;

ParanjothyS@cardiff.ac.uk

\section{ABSTRACT}

Purpose Recruitment and follow-up in epidemiological studies are time-consuming and expensive. Combining online data collection with a register of individuals who agree to be contacted about research opportunities provides an efficient, cost-effective platform for population-based research. HealthWise Wales (HWW) aims to facilitate research by recruiting a cohort of individuals who have consented to be informed about research projects, advertising studies to participants, supporting data collection on specific topics and providing access to linked healthcare data for secondary analyses. In this paper, we describe the design of the project, ongoing data collection, methods of data linkage to routine healthcare records, baseline characteristics of participants, the strengths and limitations of the register, and the ways in which the project can support researchers.

Participants Adults (aged 16 years and above) living or receiving their healthcare in Wales are eligible for inclusion. Participants consent to be contacted for followup data collection and for their details to be used to access their routinely collected National Health Service records for research purposes. Data are collected using a web-based application, with new questionnaires added every 6 months. Data collection on sociodemographic and lifestyle factors is repeated at intervals of 2-3 years. Recruitment is ongoing, with 21779 participants alive and currently registered.

Findings to date $99 \%$ of participants have complete information on age and sex, and 64\% have completed questionnaires on sociodemographic and lifestyle factors. These data can be linked with national health databases within the Secure Anonymised Information Linkage (SAIL) databank, with $93 \%$ of participants matching a record in SAIL. HWW has facilitated the recruitment of 43826 participants to 15 different studies.

Future plans The medium-term goal for the project is to enrol at least 50000 adults. Recruitment strategies are being devised to achieve a study sample that closely models the population of Wales. Potential biosampling methods are also currently being explored.

\section{INTRODUCTION}

High-income countries continue to face major public health challenges, including persistent
Strengths and limitations of this study

- More than 20000 individuals with a diverse sociodemographic profile have registered, and recruitment is ongoing.

- Matching rates of participant data with routinely collected healthcare records are very high.

- Participants are 'research ready', with HWW facilitating the recruitment of 43826 participants to 15 different studies to date.

- Men, individuals below 25 years and over the age of 65 years, and participants from the most deprived wealth quintiles are currently under-represented. Recruitment strategies to increase the number of participants in these groups are currently being devised.

- Currently, biosamples are not collected from participants, although options for this are being explored.

inequalities in health and well-being and the complex needs of ageing populations. ${ }^{12}$ Meeting these challenges requires a strong research infrastructure to ensure that highquality evidence is generated, for example, on preventing the onset and progression of non-communicable diseases and providing effective and efficient health and care services. ${ }^{3}$ Large-scale longitudinal studies are an essential resource for studying health and well-being throughout the life course. It is estimated that around $3.5 \%$ of the UK population are current or recent contributors to cohort studies. ${ }^{4}$ Using web-based technologies potentially makes recruitment and retention of subjects in such long-term studies less time-consuming and expensive. ${ }^{5}$ Combining online data collection with a register of individuals who have volunteered to be contacted with opportunities to take part in research also confers additional efficiency (such as the Scottish Health Research Register, SHARE ${ }^{6}$ ), and can create a platform to increase public 
involvement and engagement with research. Increasing awareness of the purpose of research and opportunities for participation should result in increased recruitment to research studies, better quality research to inform policy and practice, and ultimately improved population health outcomes. ${ }^{7}$

Wales has a population of over three million people, within clearly defined geographical boundaries and with relatively low levels of migration in or out. ${ }^{8}$ It faces major challenges from a postindustrial legacy of socioeconomic deprivation and a high prevalence of unhealthy behaviours. ${ }^{39}$ High-quality, population-based research in this setting has already provided important evidence for policy and practice in the UK and beyond. ${ }^{10}$ HealthWise Wales (HWW) aims to provide an integrated cost-effective platform for conducting population-based research, by:

1. Establishing a cohort of individuals who have consented to be contacted with information on research studies that they may wish to contribute to (so-called 'research-ready' individuals).

2. Collecting longitudinal data from participants on selfreported exposures and outcomes.
3. Using routinely available healthcare data through record linkage. ${ }^{11} 12$

The purpose of HWW is to facilitate and support research that will make an important contribution to shaping the health and well-being of future generations in Wales, and to help the National Health Service (NHS) in Wales plan for the future. In this paper, we describe the design of the project, ongoing data collection, methods of data linkage to routine healthcare records, baseline characteristics of participants, the strengths and limitations of the register, and the ways in which the project can support researchers.

\section{COHORT DESCRIPTION}

\section{Setting}

Recruitment into HWW is ongoing and dynamic, with individuals joining (or leaving) on a continuous basis and with varying levels of participation during their life course. Recruitment started during a pilot phase (March 2015 to February 2016), followed by a public launch on 29 February 2016. Recruitment protocols have been

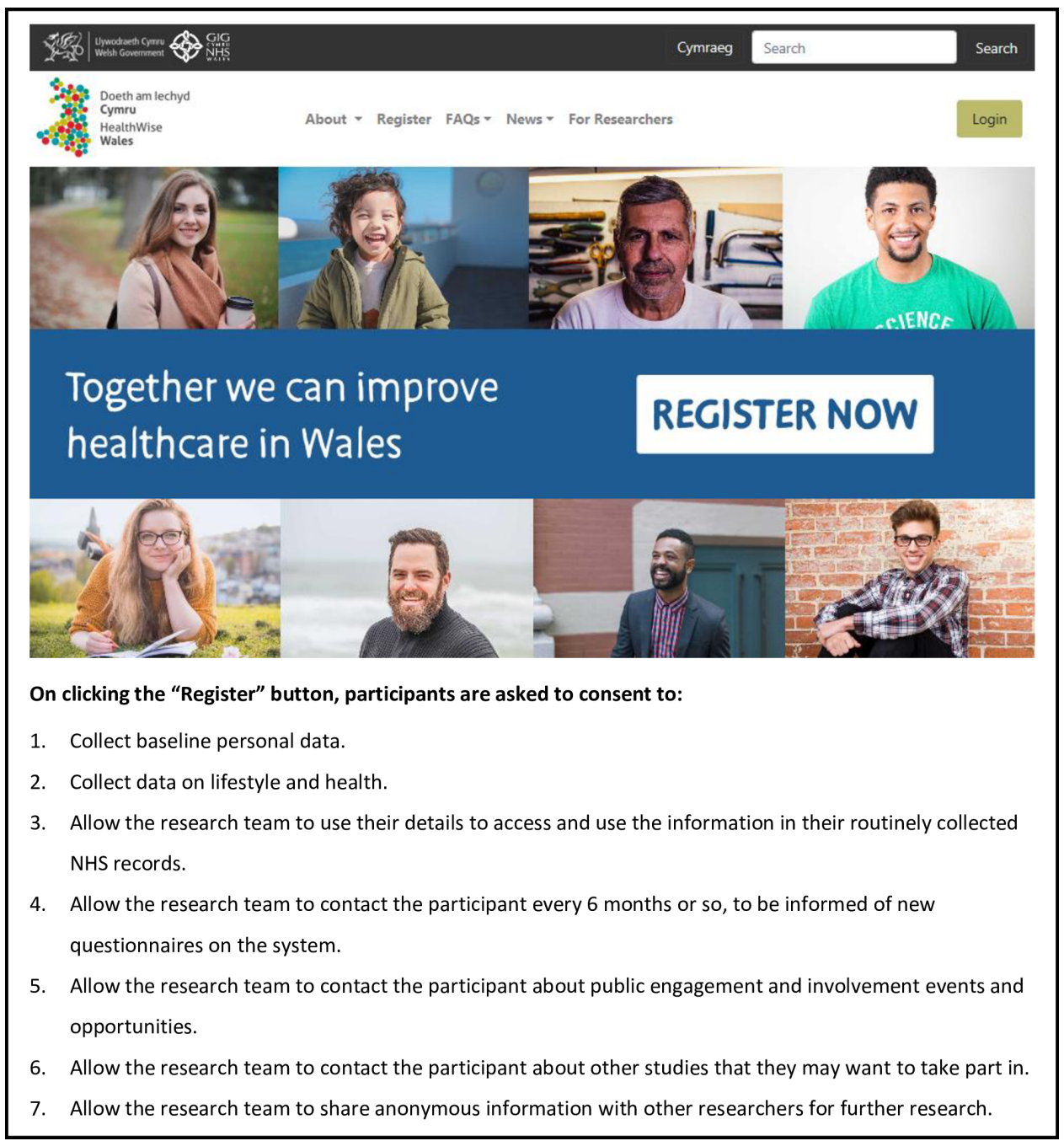

Figure 1 Website and consent. Image source: Stock images, produced by Cowshed (www.wearecowshed) on behalf of HealthWise Wales (www.healthwisewales.gov.wales) and used with permission. 


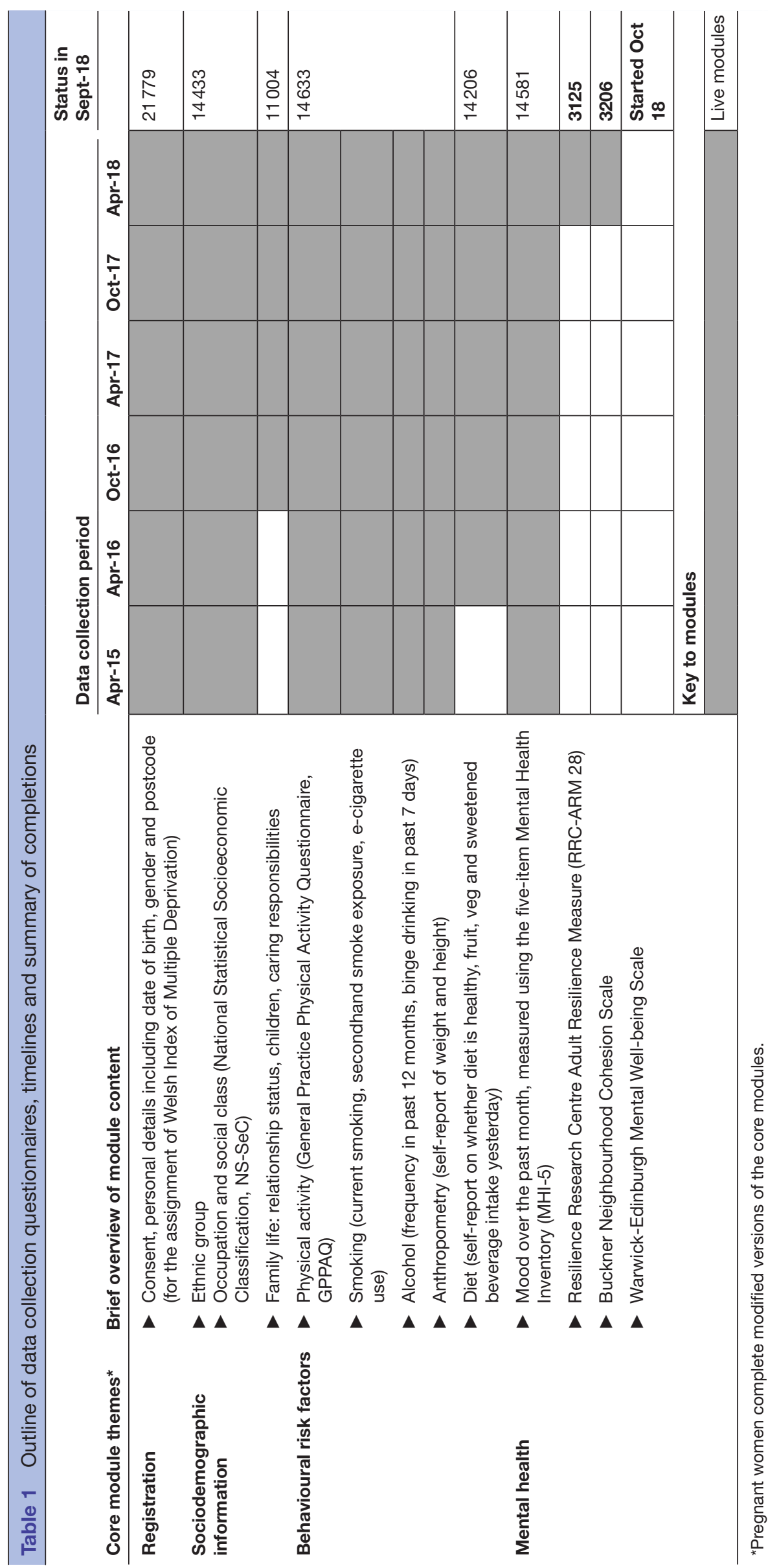

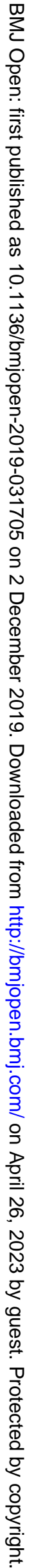




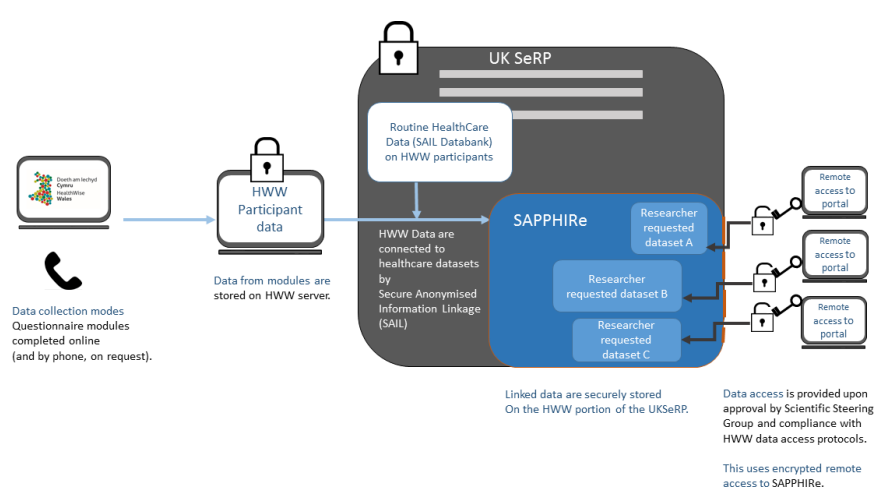

Figure 2 HealthWise Wales (HWW) data flow. SAIL, Secure Anonymised Information Linkage; SAPPHIRe, Secure Access Portal and Protected HWW Information Repository; UKSeRP, UK Secure e-Research Platform.

designed to ensure representation across all areas of Wales. Overall, the distribution of HWW participants by residence is representative of Wales. For example, census data show that $67 \%$ of the Welsh population live in urban areas (defined as settlements of at least 10000 people), ${ }^{13}$ compared with $63 \%$ in HWW.

\section{Eligibility criteria and participant recruitment}

Adults (aged 16 years or above) who are usually resident or receive their healthcare in Wales are eligible to join, and are invited to be:

1. Followed up at regular intervals to obtain information about their health, well-being and specific exposures (such as behavioural risk factors), and allow recordlinkage with their routinely collected health records.

2. Entered onto a database of potential participants for research studies.

3. Contacted to take part in specific research studies.

4. Actively engaged and involved in dialogue to shape the priorities of the research programme.

Television, radio and social media advertising campaigns have been undertaken to issue an open invitation to potential participants to register. The project has been promoted at a wide range of events across Wales (for example, cultural events such as the Eisteddfod and agricultural shows such as the Royal Welsh and Anglesey shows) and in different settings (such as NHS hospitals, general practices, pharmacy outlets and large employers). Mass postal mail-outs have also been piloted in one health board area, and there are plans to extend this method of communication about the project to other areas of Wales.

There are three core recruitment methods that are adapted for use as appropriate in different settings. First, participants can give their consent to join the project through an online web application, which is accessed via the project's website (www.healthwisewales.gov.wales, see figure 1). Second, they can be recruited face to face using tablets or paper-based sign-up forms at events and various locations across Wales. Third, they can give their consent to be contacted by individuals from the Participant Resource Centre at Cardiff University who can provide them with further information about HWW by email or telephone. Protocols describing the use of these recruitment methods and relevant study materials in various settings have been developed and have been implemented by HWW champions (members of the public who have volunteered to engage and involve other members of the public) and facilitators/research assistants (Health and Care Research Wales and NHS support and delivery staff). A range of recruitment and data collection strategies have also been developed for individuals who do not have internet access and/or may not have been exposed to the advertising campaigns. These have included study recruiters using mobile technologies with an internet connection to collect data at community-based locations, or telephone-based consent and data collection.

The medium-term goal is to enrol at least 50000 adults. This proposed sample size will be significantly larger than current population-based surveys in Wales, providing more precise estimates of the prevalence of exposures and outcomes in different sociodemographic groups, and adequate power to answer a range of different research questions about the determinants of health and well-being.

\section{Research themes}

The project has five research themes:

1. Impact of social inequalities on health and well-being.

2. Environment, neighbourhood and health.

3. Maintenance of health and well-being in the workingage population.

4. Well-being in later life.

5. Innovation in health and social care services.

These themes are broad to guide data collection and facilitate use of the HWW platform by a wide range of health and social care researchers. Across these themes, there is a focus on four health areas (cancer; mental health; dementia; and family life, pregnancy and early childhood health and development).

\section{Methods of data collection and follow-up}

Data are collected using a web-based application, designed specifically for the project, which is accessible to participants through the main HWW website. New questionnaires are added every 6 months. These either collect information on items relevant to the research themes outlined above, or bespoke data to facilitate researcher-led projects that are aligned to the research themes. Descriptive information on the core research questionnaires, their availability to participants since the project launched in 2015 and completion numbers are presented in table 1. These collect data on sociodemographic factors, lifestyle factors, home life, and mental health at baseline and will be repeated at intervals of 2-3years as appropriate. There is also an additional set of modified core questionnaires that collect information from pregnant women on their health and care.

Outcome data are obtained in two ways. First, data are collected on the HWW platform for patient-reported 
Table 2 Characteristics of the HealthWise Wales cohort and population data from published sources for Wales

\begin{tabular}{|c|c|c|}
\hline Characteristic & HealthWise Wales & Population data for Wales from published sources \\
\hline Total who have completed registration form & 21779 & \\
\hline \multicolumn{3}{|l|}{ Age group } \\
\hline $16-24$ years & $10 \%$ & $14 \% *$ \\
\hline $25-44$ years & $30 \%$ & $29 \%$ \\
\hline $45-64$ years & $38 \%$ & $32 \%$ \\
\hline $65+$ years & $22 \%$ & $25 \%$ \\
\hline Completed by (\% of those registered) & $\mathrm{n}=21746(99 \%)$ & \\
\hline \multicolumn{3}{|l|}{ Sex } \\
\hline Male & $28 \%$ & $49 \% \dagger$ \\
\hline Female & $72 \%$ & $51 \%$ \\
\hline Completed by (\% of those registered) & $\mathrm{n}=21737(99 \%)$ & \\
\hline \multicolumn{3}{|l|}{ Ethnic group } \\
\hline White & $98 \%$ & $98 \% \ddagger$ \\
\hline Other & $2 \%$ & $2 \%$ \\
\hline Completed by (\% of those registered) & $\mathrm{n}=14912(69 \%)$ & \\
\hline \multicolumn{3}{|l|}{ Standard occupational classification§ } \\
\hline 1. (Higher managerial, administrative and professional occupations) & $50 \%$ & $27 \%$ \\
\hline 2. (Intermediate occupations) & $18 \%$ & $21 \%$ \\
\hline 3. (Routine and manual occupations) & $11 \%$ & $37 \%$ \\
\hline Other (Never-worked, long-term unemployed and full-time students) & $21 \%$ & $15 \%$ \\
\hline Completed by (\% of those completing questionnaire) & $\mathrm{n}=13268(97 \%)$ & \\
\hline \multicolumn{3}{|l|}{ Physical activity (GPPAQ) } \\
\hline Active & $35 \%$ & No comparable \\
\hline Moderately active & $21 \%$ & data found \\
\hline Moderately inactive & $15 \%$ & \\
\hline Inactive & $29 \%$ & \\
\hline Completed by (\% of those completing questionnaire) & $\mathrm{n}=13647(98 \%)$ & \\
\hline \multicolumn{3}{|l|}{ Smoking } \\
\hline Current smoker & $10 \%$ & $19 \%$ ** \\
\hline Ex-smoker & $35 \%$ & $29 \%$ \\
\hline Never smoked & $55 \%$ & $52 \%$ \\
\hline Completed by (\% of those completing questionnaire) & $\mathrm{n}=13881(99 \%)$ & \\
\hline Binge drinking in past week & $23 \%$ & $24 \% \dagger †$ \\
\hline Completed by (\% of those completing questionnaire) & $n=12229(88 \%)$ & \\
\hline Drinking alcohol above guideline levels: & $50 \%$ & $40 \% \dagger \dagger$ \\
\hline Completed by (\% of those completing questionnaire) & $n=12229(88 \%)$ & \\
\hline \multicolumn{3}{|l|}{ Mental Health Inventory (MHI-5) score } \\
\hline Score consistent with common mental disorder $\neq \ddagger$ & $28 \%$ & \\
\hline Completed by (\% of those completing questionnaire) & $n=13694(99 \%)$ & \\
\hline Diagnosed with or treated for a mental health condition? & $32 \%$ & $13 \% \S \S$ \\
\hline Completed by (\% of those completing questionnaire) & $\mathrm{n}=13837$ (99\%) & \\
\hline
\end{tabular}

*Mid-year population estimates for Wales 2017, Office for National Statistics (from StatsWales, https://statswales.gov.wales/).

†Mid-year population estimates for individuals aged 16+ years in Wales 2017, Office for National Statistics (from StatsWales).

†Population estimates for individuals aged 16+ years in Wales 2011, Office for National Statistics (from StatsWales).

§Using the National Statistics Socioeconomic Classification (NS-SEC, Office for National Statistics).

\Population estimates for individuals aged 16-74 years in Wales, 2011 UK Census.

**Estimates for individuals 16+ years from the National Survey for Wales 2017-2018 (https://gov.wales/statistics-and-research/national-survey/?tab=el_home\&topic=population_ health\&lang=en).

t†Data for individuals aged 16+ years from the Welsh Health Survey 2015

łłKelly MJ, Dunstan FD, Lloyd K and Fone D. (2008) Evaluating cut-points for the MHI-5 and MCS using the GHQ-12: a comparison of five different methods. BMC Psychiatry 2008, 8:10

$\S \S$ Data for individuals aged 16+ years from the Welsh Health Survey 2015; asked whether they were currently being treated for 'depression, anxiety or another mental health illness'. BMC, BioMedCentral; GHQ-12, General Health Questionnaire; GPPAQ, General Practice Physical Activity Questionnaire; MCS, Mental Health Component Summary Score; MHI-5,

Five-item Mental Health Inventory; NIHR, National Institute for Health Research. 


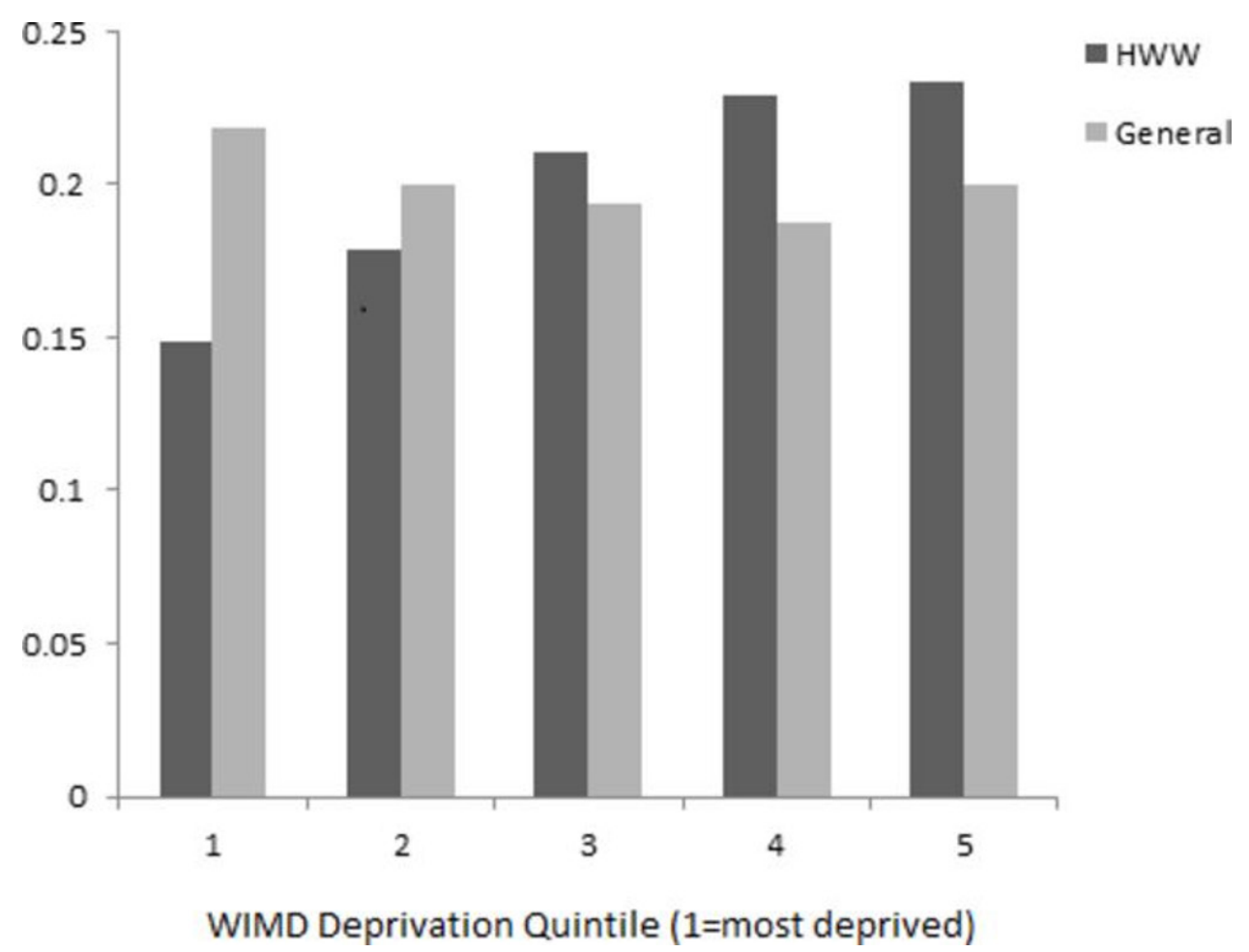

Figure 3 Proportion of participants resident in each quintile of the Welsh Index of Multiple Deprivation (WIMD), compared with the general Welsh population. HWW, HealthWise Wales.

outcome measures and those relevant to conditions likely to be under-represented in routinely collected data (for example, infections, metabolic diseases, psychiatric conditions and well-being). Second, outcome information can be obtained through record linkage with national health databases (such as the Patient Episode Database for Wales and general practice data) within the Secure Anonymised Information Linkage (SAIL) databank. ${ }^{11}{ }^{12}$ Future phases of the project will also include linkage with other administrative data sets.

The NHS Wales Informatics Service (a trusted third party) uses the personal details of participants (with their consent) to generate an anonymised linking field based on their name, address, gender and date of birth. This is used to link participants' data with routinely collected healthcare data sets, with $93 \%$ of active participants matching with a record in SAIL. The SAIL databank and the Secure Access Portal and Protected HWW Information Repository (SAPPHIRe) are stored in separate areas of the UK Secure e-Research Platform (UKSeRP, ${ }^{14}$ ). Figure 2 shows the flow of project data, showing SAPPHIRe within UKSeRP where project-specific, anonymised HWW data can be accessed.

\section{Patient and public involvement}

HWW has a specific focus on increasing public involvement and engagement in health and social care research. To ensure these aims are achieved, the project is overseen by a Public Involvement Delivery Board (PIDB), which is chaired by an independent member of the public and whose membership is predominantly comprised of members of the public. The PIDB provides scrutiny and assurance that the project is operating in the public interest, and provides advice and support in delivering best practice in accordance with the National Standards for Public Involvement (https://www.invo.org.uk/posttypepublication/nationalstandards-for-public-involvement/). The Board and the research team co-produced the project's patient and public involvement (PPI) policy and implementation plan. The research team has a dedicated PPI lead who is responsible for maintaining the policy document and ensuring compliance with it. All research team members are trained on facilitating public involvement. There are two PPI members of the research team, who have agreed objectives for their role and attend monthly meetings where they are actively involved in discussions and decision-making relating to research team activities. We have also trained 78 local health board members as facilitators to engage the public and recruit participants to HWW.

Involvement opportunities (including participation in media promotions or development and user-testing of data collection questionnaires) are regularly offered to participants through a quarterly e-newsletter. As a result, three participants became the faces of the advertising campaign in March 2017, others have participated in social media promotions, and 156 agreed to be members of a user-testing panel.

PPI is an essential criterion for all studies that use HWW, and researchers are required to describe the PPI they have undertaken when applying to use the data or the platform. PPI research team members scrutinise this element of applications as part of their overall assessment of all new projects. 
Table 3 Engagement of HealthWise Wales (HWW) participants with research advertised via the HWW platform

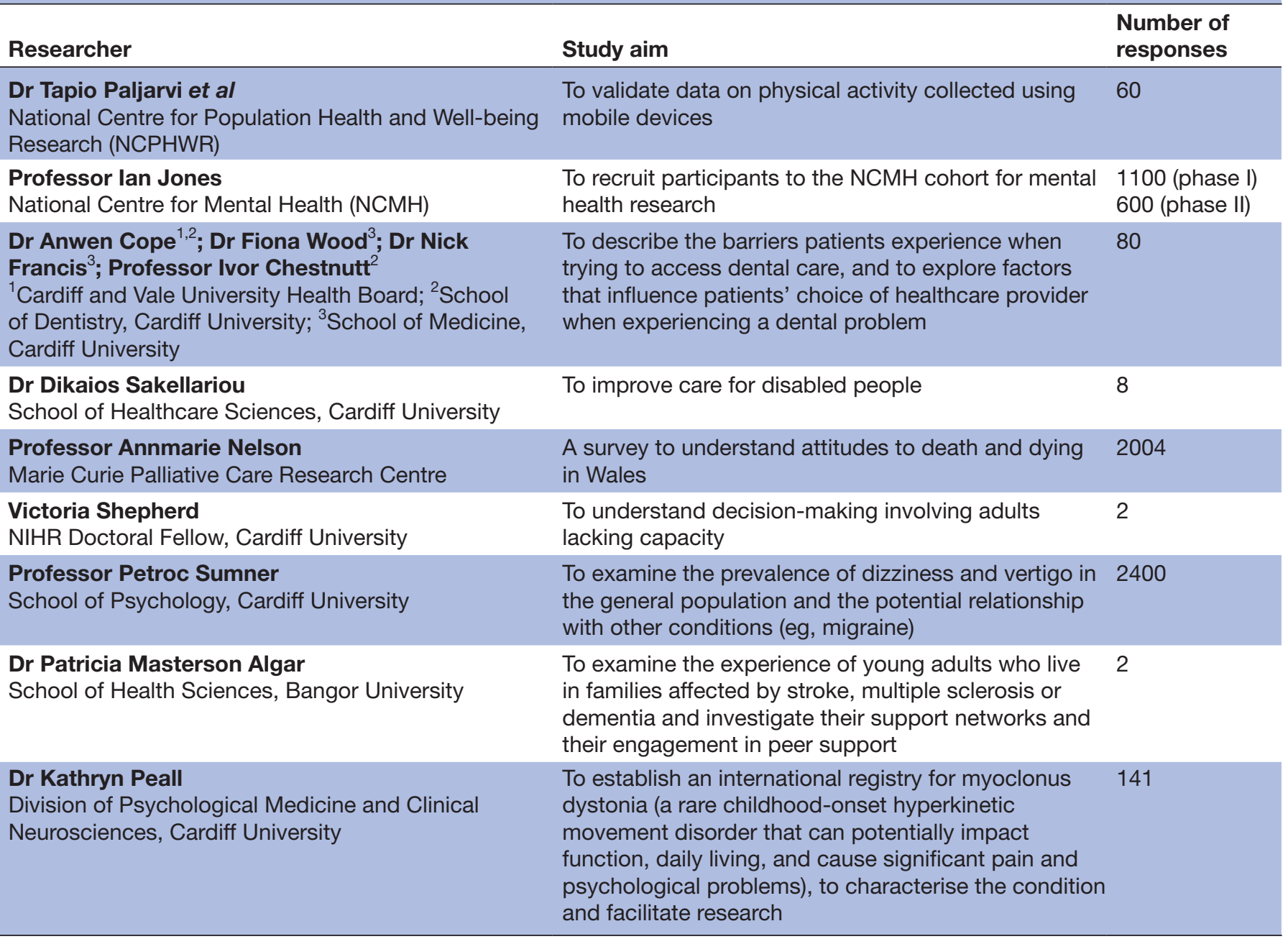

\section{Ethical approval and governance arrangements}

The project is overseen by an executive group, which provides oversight and decision making on the overall delivery of initiative, and receives advice from a Scientific Steering Group (SSG) and the PIDB. The role of the PIDB has been described above.

HWW received ethical approval from WalesResearch Ethics Committee (REC) 3 on 16th March 2015 (reference 15/WA/0076). Substantial amendments are submitted when new questionnaires are added or if there is a substantial change to the content of participant-facing materials or recruitment model, in line with current guidance from the committee. The data collection system and study processes are designed to safeguard the integrity and confidentiality of data collected and generated for HWW research, and appropriate systems have been established and tested to report any failures in these respects. Standard operating procedures are in place to ensure that HWW is conducted within research governance regulations and is compliant with the General Data Protection Regulation (EU) 2016/679. The research team meet with the HWW Data Guardian every 6 months to review the data governance processes in place and any matters arising.

\section{FINDINGS TO DATE}

There are currently more than 20000 active participants (alive and currently registered). Ninety-nine per cent of registered participants have complete information on age and sex, and at least $64 \%$ have completed the other core questionnaires. Table 2 shows the characteristics of active participants compared with data from published sources of Welsh data. Compared with the population of Wales, there is a higher percentage of participants who are 45-64 years old. The percentage of women is higher than in the general population $(72 \%$ compared with $51 \%)$. The percentage of participants in non-white ethnic groups $(2 \%)$ is the same as in the general population. Fifty per cent of participants are classified as being in higher managerial or professional occupations, compared with $27 \%$ of the population of Wales. In terms of healthrelated behaviours: $56 \%$ are classified as active or moderately active; $10 \%$ are current smokers (compared with $19 \%$ of the general population); and $50 \%$ drink more alcohol than recommended by UK guidance (compared with $40 \%$ of the general population). Twenty-eight per cent of participants have a Mental Health Inventory Score consistent with a common mental disorder and $32 \%$ have been diagnosed with or treated for a mental 


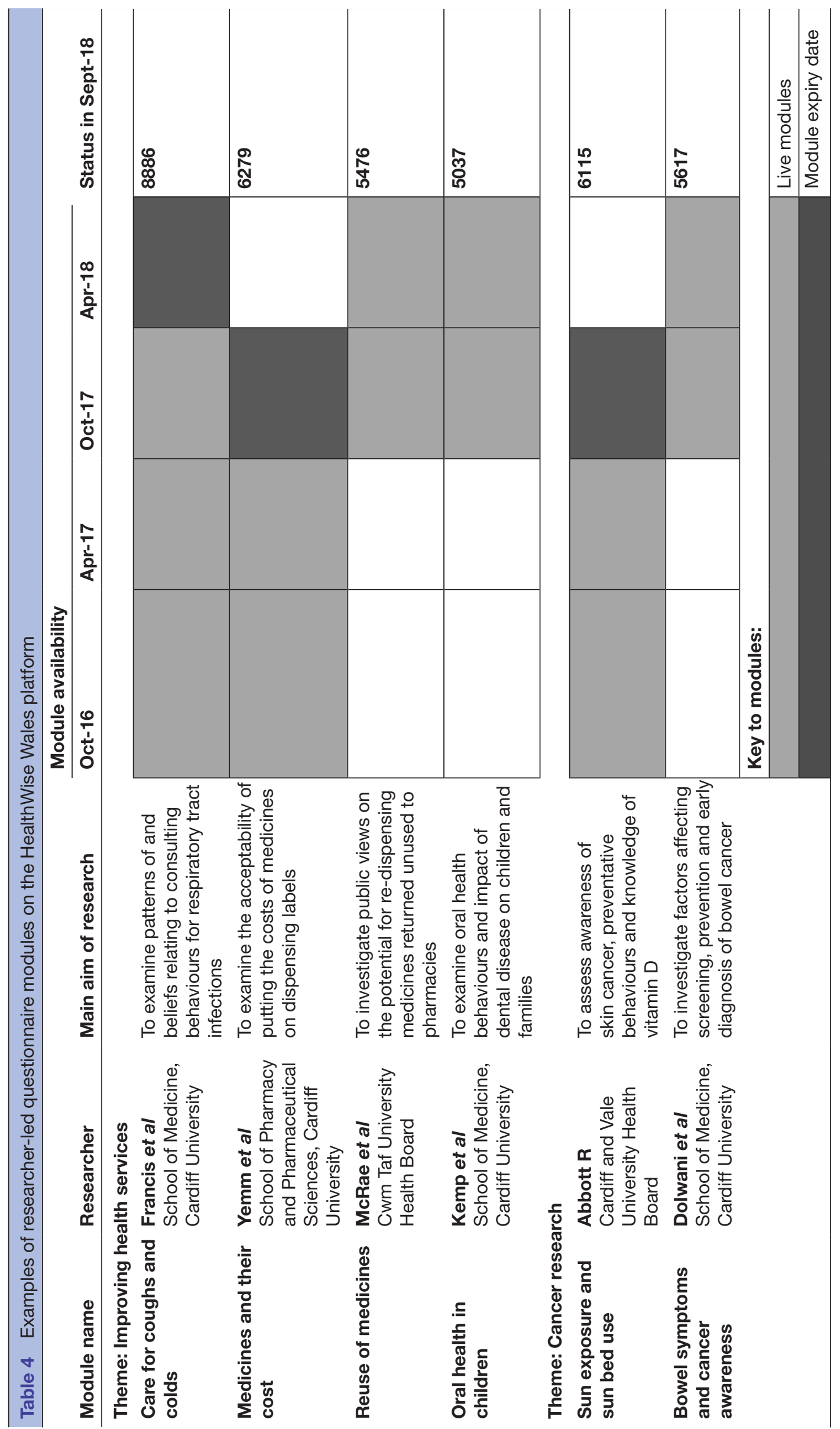


Researcher contacts HWW to discuss possible project

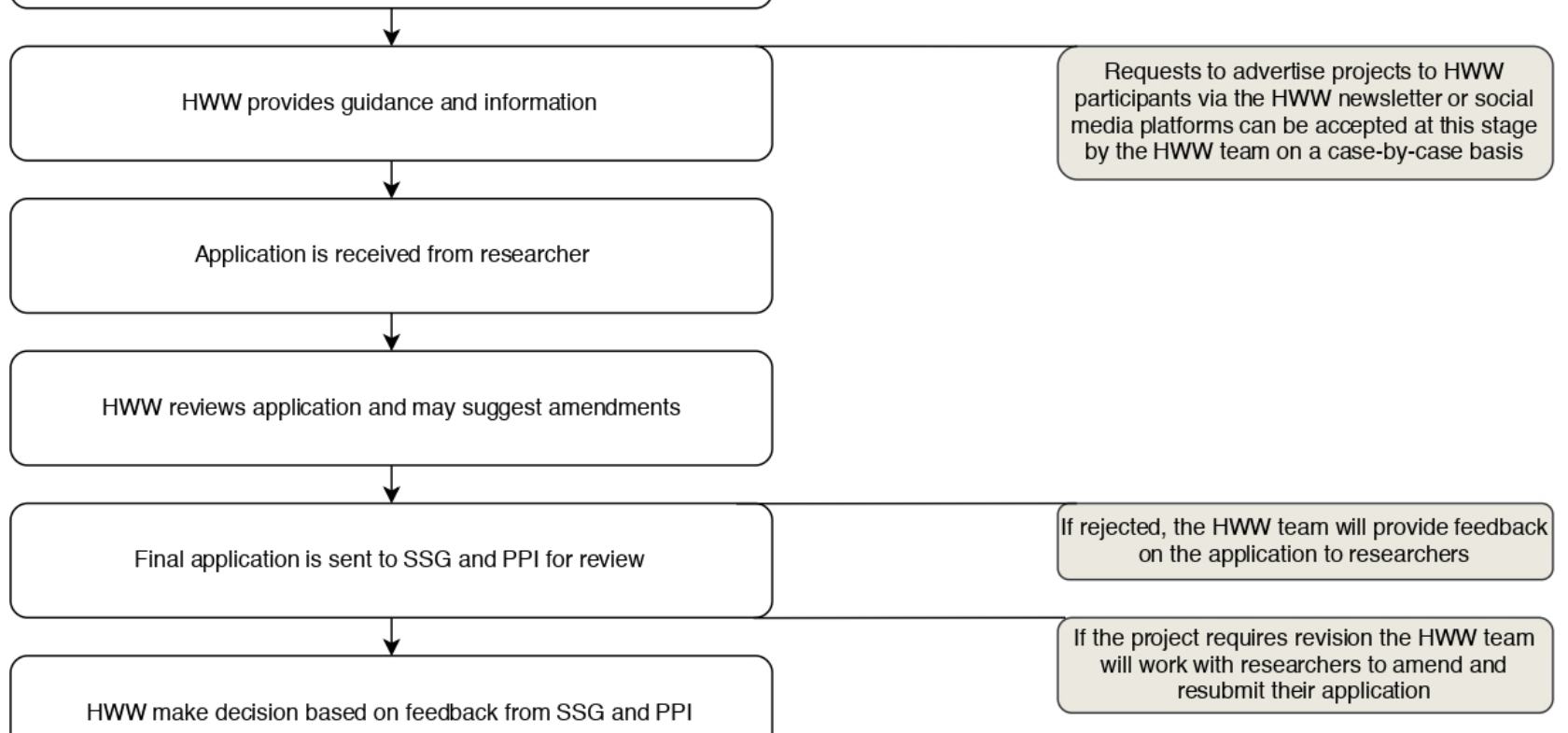

HWW make decision based on feedback from SSG and PPI

Project approved

HWW Participant Sample

HWW team send targeted

advertisement to identified participants
HWW Linked Data

Researchers provide evidence

of compliance with

Information Govemance

HWW provide access to HWW

linked data via SAPPHIRe

For all types of study you can apply at any time

Expect a response to your application within 10 working days
SSG meet biannually in April and October. Expect a response within one month of meeting

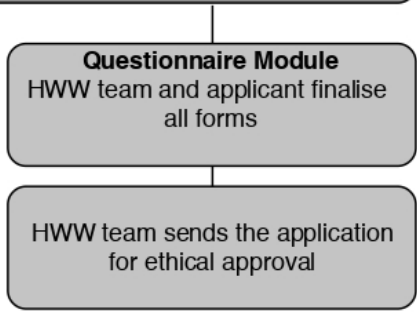

\section{Timelines}

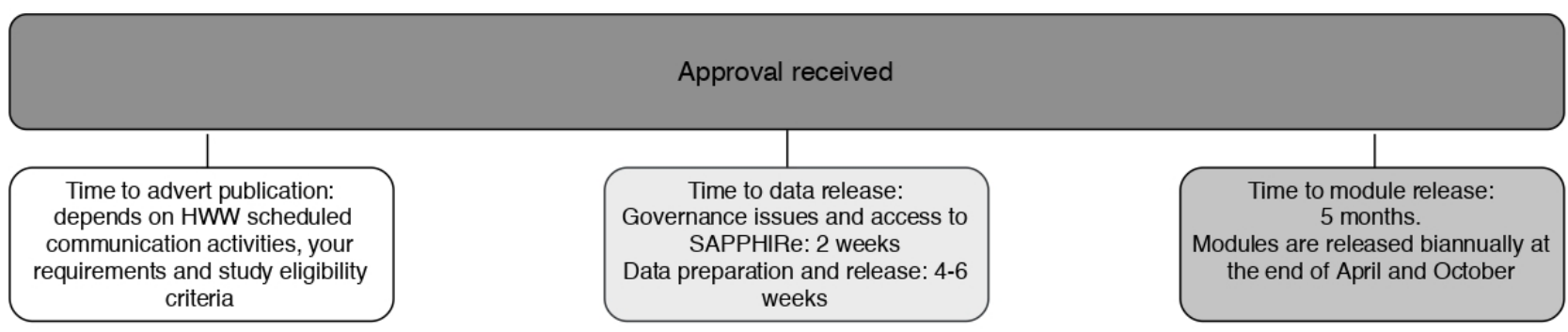

Charges for the use of the HWW platform will be determined on a case by case basis and will be applied to any application approved/actioned after 1 April 2019. Contact the HWW team to discuss.

HWW HealthWise Wales, PPI Patient and Public Involvement, SSG Scientific Steering Group, SAPPHIRe Secure Anonymous Portal and Protected HWW Information Repository

Figure 4 Flow diagram showing the application process for all HWW activities. 
health condition (compared with 13\% of the general population). Figure 3 shows the distribution of participants according to the Welsh Index of Multiple Deprivation compared with the population of Wales. There is a good representation of participants in each deprivation quintile, although a higher percentage of participants is from the least deprived quintile.

\section{RESEARCH ACTIVITIES}

HWW supports researchers in three ways by: advertising relevant studies to participants; providing access to cohort data for secondary analyses via the researcher portal; and supporting data collection on specific topics within the platform that can then be linked with healthcare data. To date, seven studies have used the database to inform potential participants of an opportunity to take part in their research (see table 3), with recruitment for each of these exceeding the required target. Nine studies have used the platform to collect data on study-specific questionnaires (see table 4), with more than 5000 participants providing data for each of these. In total, HWW has facilitated the recruitment of 43826 participants to 15 different studies to date, with many of the 21779 registrants taking part in multiple studies. Results from these studies are now being published including, for example, an analysis of patients' reasons for consulting a general practitioner when they had a dental problem. ${ }^{15}$ Links to all publications that have used HWW to recruit participants will be included on the project website.

\section{STRENGTHS AND LIMITATIONS}

There are several strengths of HWW as a resource for research. More than 20000 individuals with a diverse sociodemographic profile have already registered, and recruitment is ongoing. Matching rates of participant data with routinely collected healthcare records are very high. In contrast with other population-based cohorts in the UK, ${ }^{4} \mathrm{HWW}$ participants are younger, with most between 30 years and 60 years old. This provides an opportunity to conduct longitudinal population studies with data collected predisease onset. Participants are also 'research ready'; the examples given above demonstrate that the platform provides an effective way for the research community to reach an engaged, responsive cohort. A targeted retention plan is being developed with PPI representatives and a wider stakeholder group to encourage continued active participation in the project. Strategies found by other studies to be effective will be adapted to suit the HWW cohort, including the provision of real-time feedback to participants when they provide data, the development of an online community where participants can share their research experiences, and regular, diverse public engagement events to disseminate emerging results.

Men are currently under-represented in the cohort; only $28 \%$ of registered participants are male. Similarly, there are fewer individuals below 25 years and over the age of 65 years than in the general population, and a smaller percentage of participants from routine and manual occupations and in the most deprived wealth quintiles. To address this, we are currently undertaking qualitative research using a stratified sampling frame (based on age and gender) in deprived areas of Wales. We are recruiting engaged and disengaged participants with the aim of understanding motivations and barriers to participating in HWW and collecting suggestions from them for future communications, marketing and interactive activities that would appeal to under-represented segments of the population. Focus group participants will be asked to comment on recruitment strategies that are currently being considered including arts-based workshops, and celebrity and local ambassador programmes. Our retention and recruitment strategies will inform and reinforce each other. A key feature of both will be participant involvement in design and development of these activities to promote a two-way, dynamic flow of information between the research team, participants and members of the public to encourage greater public involvement with research. The aim is to achieve a study sample that closely models the population of Wales, with sufficient numbers in sociodemographic subgroups to allow for the selection of populations for research from those groups. For example, the cohort currently includes 5000 men, providing a substantial sample size that will be adequate for some analyses.

Research registers, such as SHARE Scotland, ${ }^{6}$ are increasingly recognised as an effective and efficient way of supporting recruitment for research. Not all registers operate in the same way. For example, SHARE Scotland uses information from the NHS records of their registrants to determine their suitability for individual projects. HWW plans to offer this option to researchers in future. SHARE Scotland does not collect self-reported exposure and outcome information from participants, whereas HWW does. Lastly, SHARE Scotland gives participants the opportunity to consent to the storage and use of 'spare' blood (blood remaining from tests taken within the NHS), which can then be used in approved medical research. Currently, biosamples are not collected from HWW participants. Formative research examining the willingness of individuals to provide different types of biological samples for research as part of their participation in HWW showed that $83 \%$ would be willing to do so. Options for a strategic approach to biosampling across Wales, and therefore a future enhancement that will increase the value of this cohort, are currently being explored.

\section{COLLABORATION}

Figure 4 shows the application process for all research activities that can be undertaken using the HWW platform. All documentation informing researchers of how to apply to use the HWW platform was made public 
in June 2018, and access to the data has been possible since September 2018. A guide for researchers giving full details of the application and review process, and a copy of the application form, are available on the study website (www.healthwisewales.gov.wales/for-researchers). Requests to advertise projects to HWW participants via newsletters or social media are reviewed on a case-bycase basis by the HWW research team. The HWW ethical approval and participant consent permit HWW to advertise research projects to registered participants as long as they fit with the ethos and scope of the initiative. It is the responsibility of applicants to obtain ethical approval for the conduct of their specific study before HWW advertises it to participants. This ethical approval should specify that HWW will be used to help recruit participants.

Applications to use HWW for data collection or analysis are reviewed by the SSG and by PPI representatives, to assess that the project fits with the ethos of HWW, is scientifically sound and that adequate PPI input has been sought in the development of the proposal. Once approved, researchers work closely with the HWW research team to deliver the project, including working together to prepare the application for a substantial amendment to the HWW ethical approval (which is needed for all new data collection). Researchers will need to provide evidence that they are bona fide researchers and have appropriate training in research data and confidentiality procedures in order to gain access to the HWW data repository via SAPPHIRe.

Further details on how to apply, and the requirements for access and acknowledgements for publication, can be found in the Researcher Guidance document on the Researcher tab of the HWW website: www.healthwisewales.gov.wales/for-researchers/. Researchers should contact the research team (on healthwisewales@cardiff. ac.uk or 08009172172 ) before submitting their application to obtain guidance on how best to use the platform in their study, PPI processes, ethical requirements, questionnaire development, implementation and promotion.

In conclusion, HWW is a research database of adults (aged 16 years and above) living or receiving their healthcare in Wales that can support researchers by: advertising relevant studies to registered participants; providing access to cohort data for secondary analyses via the researcher portal; and supporting data collection on specific topics with record linkage to healthcare data if required. It has been successful in recruiting a 'research ready' cohort in Wales, and to date has facilitated recruitment of 43826 participants into 15 studies.

\section{Twitter Lisa Hurt @healthwisewales?lang=en}

Acknowledgements The authors thank Charlotte Bonner-Evans and Ameeta Richardson for their contribution in the coordination, management and implementation of the platform. The authors also thank Sean Dunn, Benjamin Dowie, Alex Coomber and others within the Participant Resource Centre (Cardiff University) and the Health and Care Research Wales Support and Delivery Centre for their contribution to recruitment and data collection, and the Welsh Government Communication team for their contribution to the Communications Plan. The authors also thank the Scientific Steering Group, the HealthWise Wales Executive group, the Public Involvement Delivery Board, Chris Stock, the Centre for Trials Research and Professor Mike Robling (Data Guardian) for substantial contribution. The authors also thank the participants.

Contributors SP is the principal investigator of HealthWise Wales. LHu, PA-W, JT, $\mathrm{LH}, \mathrm{LC}, \mathrm{MDA}, \mathrm{JH}$ and SP contributed to the conceptualisation of the project, data collection and analysis methods, and recruitment and retention methods. LHu and SP wrote the first draft of this paper. PA-W, JT, LHe, LC, MDA, JH and SP contributed to the text of the paper, subsequent revisions and the production of the final version of the paper.

Funding HealthWise Wales is funded by Health and Care Research Wales.

Competing interests None declared.

Patient consent for publication Not required.

Ethics approval HWW received ethical approval from the Wales Research Ethics Committee (REC) 3 on 16 March 2015 (reference 15/WA/0076).

Provenance and peer review Not commissioned; externally peer reviewed. Data availability statement Data are available upon reasonable request.

Open access This is an open access article distributed in accordance with the Creative Commons Attribution Non Commercial (CC BY-NC 4.0) license, which permits others to distribute, remix, adapt, build upon this work non-commercially, and license their derivative works on different terms, provided the original work is properly cited, appropriate credit is given, any changes made indicated, and the use is non-commercial. See: http://creativecommons.org/licenses/by-nc/4.0/.

ORCID iD

Lisa Hurt http://orcid.org/0000-0002-2741-5383

\section{REFERENCES}

1 Marmot M. The health gap. The challenge of an unequal world London. Bloomsbury Publishing, 2015.

2 Prince MJ, Wu F, Guo Y, et al. The burden of disease in older people and implications for health policy and practice. The Lancet 2015;385:549-62.

3 Chief Medical Officer. Chief medical officer for Wales annual report 2015-16. rebalancing healthcare. working in partnership to reduce social inequity. Cardiff: Welsh Government, 2016.

4 Medical Research Council. Maximising the value of UK population cohorts. MRC strategic review of the largest UK population cohort studies. London: Medical Research Council, 2014.

5 Manolio TA, Weis BK, Cowie CC, et al. New models for large prospective studies: is there a better way? Am J Epidemiol 2012;175:859-66.

6 McKinstry B, Sullivan FM, Vasishta S, et al. Cohort profile: the Scottish research register share. A register of people interested in research participation linked to NHS data sets. BMJ Open 2017;7:e013351.

7 Staley K. 'Is it worth doing?' measuring the impact of patient and public involvement in research. Res Involv Engagem 2015;1:6.

8 Office for National Statistics. Statistical Bulletin. Population estimates for the UK, England and Wales, Scotland and Northern Ireland: mid2017. London: Office for National Statistics, 2018.

9 Chief Medical Officer. Chief medical officer for Wales annual report 2014-15. Healthier, happier, fairer Cardiff: Wesh Government, 2015.

10 Elwood P, Galante J, Pickering J, et al. Healthy lifestyles reduce the incidence of chronic diseases and dementia: evidence from the Caerphilly cohort study. PLoS One 2013;8:e81877.

11 Ford DV, Jones KH, Verplancke J-P, et al. The Sail Databank: building a national architecture for e-health research and evaluation. BMC Health Serv Res 2009;9:157.

12 Lyons RA, Jones KH, John G, et al. The Sail databank: linking multiple health and social care datasets. BMC Med Inform Decis Mak 2009;9:3.

13 Office for National Statistics. 2011 Census Analysis - Comparing Rural and Urban Areas of England and Wales. London: Office for National Statistics, 2013.

14 Jones KH, Ford DV, Jones C, et al. A case study of the secure anonymous information linkage (Sail) gateway: a privacy-protecting remote access system for health-related research and evaluation. $J$ Biomed Inform 2014;50:196-204.

15 Cope AL, Wood F, Francis NA, et al. Patients' reasons for consulting a GP when experiencing a dental problem: a qualitative study. $\mathrm{Br} J$ Gen Pract 2018;68:e877-83. 